\title{
Utilidad de la laparoscopia de estadificación frente a la tomografía axial computarizada para detectar metástasis peritoneales en el adenocarcinoma gástrico avanzado
}

\author{
Usefulness of staging laparoscopy vs CT scan to detect metastatic \\ peritoneal disease in advanced gastric adenocarcinoma \\ José Luis Espinosa1, Liliana María Suárez², Raúl Enrique Guevara², \\ Fredy Orlando Mendivelso 3
}

\footnotetext{
Médico, residente de Cirugía, Fundación Universitaria Sanitas, Bogotá, D.C., Colombia

2 Médico cirujano, Servicio de Cirugía Gastrointestinal, Clínica Universitaria Colombia, Bogotá, D.C., Colombia

3 Médico, epidemiólogo clínico, Clínica Reina Sofía, Bogotá, D.C., Colombia
}

\section{Resumen}

Introducción. El adenocarcinoma gástrico es una de las neoplasias más frecuentes. La tomografía computarizada abdominal con contraste es el método estándar para la estadificación; tiene una sensibilidad del 30 al 73 \% y una especificidad del 83 al Ioo \%. La laparoscopia detecta hasta el $30 \%$ de los casos de enfermedad metastásica que no se haya observado en los estudios de imágenes. En la Clínica Universitaria Colombia se realiza estadificación rutinaria con tomografía y laparoscopia más lavado peritoneal.

Objetivos. Determinar la utilidad de la laparoscopia para detectar la carcinomatosis peritoneal en los pacientes con adenocarcinoma gástrico avanzado en un centro de referencia.

Materiales y métodos. Se llevó a cabo un estudio descriptivo y retrospectivo, en el cual se analizaron todas las historias clínicas de los pacientes con cáncer gástrico atendidos en la Clínica Universitaria Colombia entre el 2013 y el 20I6. Se confrontó el hallazgo de la laparoscopia con el de la tomografía abdominal, buscando falsos negativos para la enfermedad peritoneal.

Resultados. Se incluyeron 94 pacientes con adenocarcinoma gástrico. La localización en el cuerpo gástrico fue la más frecuente (47,9\%). La tomografía reportó estadio $\mathrm{T}_{3}$ en el 56,4 \%, $\mathrm{N}_{\mathrm{o}}$ en el 55,3\% y $\mathrm{M}_{\mathrm{o}}$ en el 97,9\% de los casos. La laparoscopia reportó estadio $\mathrm{T}_{3}$ en el 43,6 \%, ganglios comprometidos en el 56,4 \% y carcinomatosis peritoneal ( $\mathrm{M}_{\mathrm{r}}$ ) en el II, $7 \%$.

Discusión. Un radiólogo experimentado detecta una gran proporción de las enfermedades peritoneales, pero la laparoscopia detecta hasta II \% de aquellas que no son evidentes en los exámenes de imágenes. El lavado peritoneal no prolonga el tiempo quirúrgico ni incrementa la morbilidad, lo cual favorece su realización rutinaria.

Fecha de recibido: 17/01/2019 - Fecha de aceptación: 7/05/2019

Correspondencia: José Luis Espinosa, MD, Carrera 70B Nº64D-35, apartamento 201, Bogotá, D.C., Colombia. Teléfono: (304) 376-6186. cirugiageneralcuc@colsanitas.com

Citar como: Espinosa JL, Suárez LM, Guevara RE, Mendivelso FO. Utilidad de la laparoscopia de estadificación frente a la tomografía axial computarizada para detectar metástasis peritoneales en el adenocarcinoma gástrico avanzado. Rev Colomb Cir. 2019;34:254-9. https://doi.org/10.30944/20117582.439

Este es un artículo de acceso abierto bajo una Licencia Creative Commons - BY-NC-ND https://creativecommons.org/licenses/by-nc-nd/4.0/deed.es 
Se puede recomendar la laparoscopia en aquellos pacientes con resultados tomográficos negativos, evitando la cirugía cuando la neoplasia es irresecable. La laparoscopia tiene un impacto positivo en el manejo integral del cáncer gástrico, acorde con la literatura mundial.

Palabras clave: neoplasias gástricas; peritoneo; laparoscopía; tomografía; diagnóstico por imagen; oncología quirúrgica; estadificación de neoplasias

\begin{abstract}
Introduction: Gastric adenocarcinoma is one of the most frequent neoplasms. Abdominal computed tomography is the standard for staging; It has sensitivity of 30 to $73 \%$ and specificity of 83 to I00\%. Laparoscopy detects up to $30 \%$ of negative metastatic disease in images. At Clínica Universitaria Colombia performs routine staging with tomography and laparoscopy plus peritoneal lavage.

Objectives: To determine the usefulness of laparoscopy for the detection of peritoneal carcinomatosis in patients with advanced gastric adenocarcinoma in a reference center

Materials and methods: A retrospective descriptive study was carried out, where all the clinical histories of patients with gastric cancer between 2013 and 2016 were analyzed.

Results: A total of 94 patients with gastric adenocarcinoma were included in the study; Corporal localization was more frequent (47.9\%). The tomography reported stage T3 in $56.4 \%$, No in $55.3 \%$ and Mo $97.9 \%$. Laparoscopy reported $\mathrm{T} 3$ stage in $43.6 \%$, lymph nodes were involved in $56.4 \%$ and the presence of peritoneal carcinomatosis (MI) was II.7\%.

Discussion: An experienced radiologist shows high detection of peritoneal disease, but laparoscopy detects up to II\% of the disease not evident on images. The peritoneal lavage does not prolong the surgical time nor increases morbidity, which favors its routine performance. We can recommend laparoscopy in patients with negative tomography, avoiding surgery in unresectable disease. Laparoscopy has a positive impact on the management of gastric cancer, according to world literature.
\end{abstract}

Key words: stomach neoplasms; peritoneum; laparoscopy; tomography; diagnostic imaging; surgical oncology; neoplasm staging.

\section{Introducción}

El adenocarcinoma gástrico es una de las neoplasias más comunes en el mundo y causa anualmente cerca de 738.0oo muertes. En el mundo occidental, más del $50 \%$ de los casos se diagnostica en los estadios avanzados, con supervivencia global a cinco años de alrededor del 30,4 \%, incidencia anual de 7,4 casos por cada 100.000 habitantes y mortalidad de 3,3 por cada Ioo.ooo habitantes ${ }^{\mathrm{I}, 2}$.

En Colombia, en el 20I4, este tumor se consolidó como el tercero en frecuencia, con una incidencia de 7,I por Ioo.00o habitantes en mujeres y de I6,7 por I00.00o habitantes en hombres. La supervivencia a cinco años está directamente relacionada con el estadio detectado en el mo- mento del diagnóstico, y se han reportado cifras de $66,9 \%$ para el estadio I, de $30,9 \%$ para los estadios II y III, de $5 \%$ para el estadio IV y de $21,9 \%$ en los casos sin estadificar ${ }^{3,4}$.

A nivel mundial, la tomografía computarizada (TC) abdominal con contraste es el método estándar para la estadificación inicial; tiene una sensibilidad del 30 al $73 \%$ y una especificidad del 83 al Ioo \% ${ }^{2,5}$. La laparoscopia diagnóstica aún es motivo de controversia en cuanto a su uso rutinario; no obstante, se ha demostrado su utilidad para detectar la enfermedad metastásica cuando esta no es evidente en las imágenes diagnósticas ${ }^{6}$. Se ha llegado a documentar que hasta el $30 \%$ de los casos de enfermedad metastásica pueden pasarse por alto con los estudios imagenológicos ${ }^{7}$. 
Actualmente en la Clínica Universitaria Colombia, la estadificación prequirúrgica se realiza en todos los pacientes con cáncer gástrico localmente avanzado con TC abdominal con contraste y laparoscopia diagnóstica con lavado peritoneal; es el método por medio del cual se decide el tratamiento definitivo. El pronóstico global del cáncer gástrico avanzado es pobre, con una tasa de supervivencia a cinco años de 25,7\%. La mediana de supervivencia de la enfermedad metastásica o no resecable es, aproximadamente, de 8 a Io meses $^{8}$.

El objetivo de este estudio fue determinar la utilidad de la laparoscopia diagnóstica para la detección prequirúrgica de la carcinomatosis peritoneal en pacientes con adenocarcinoma gástrico avanzado, en un centro de referencia de tratamiento.

\section{Materiales y métodos}

\section{Diseño}

Se llevó a cabo un estudio descriptivo y retrospectivo.

\section{Población y fuentes}

Se analizaron todas las historias clínicas y los registros de cirugía, de los pacientes adultos de ambos sexos que fueron valorados por adenocarcinoma gástrico avanzado durante los años 2013 a 2016 en la Clínica Universitaria Colombia. Se recolectó información de las variables demográficas y clínicas, los procedimientos de cirugía, y los exámenes de laboratorio e imágenes diagnósticas.

\section{Análisis estadístico}

Se trabajó con una muestra no probabilística (o por conveniencia) y las unidades de observación se recolectaron de forma consecutiva, previa validación y cumplimiento de los criterios de inclusión.

Se incluyeron los pacientes con cáncer gástrico avanzado, cuyo manejo previo y posterior a la cirugía correspondió al Departamento de Cirugía; se hizo especial énfasis en los pacientes que habían cumplido con la recomendación de practicarse una TC abdominal con contraste prequirúrgica y, posteriormente, una laparoscopia para la estadificación.

Los datos fueron consignados en hojas de cálculo para su análisis posterior. Se calcularon las medidas de frecuencia y de tendencia central para las variables continuas, y la evaluación de su distribución de normalidad se hizo mediante los gráficos P-P y Q-Q, y la prueba de ShapiroWilks. Las variables categóricas se analizaron mediante frecuencias relativas y se calcularon los intervalos de confianza del $95 \%$ para cada una de las estimaciones.

La diferencia de los promedios de las variables relevantes se estableció con la prueba no paramétrica U de Mann-Whitney. Los valores de $\mathrm{p}$ menores de 0,05 se consideraron estadísticamente significativos. Los datos se analizaron con el software licenciado Stata ${ }^{\mathrm{TM}}$, versión I3.0.

\section{Aspectos éticos}

Este estudio correspondió a una investigación sin riesgo de acuerdo con la Resolución $\mathrm{N}^{\circ} 08430$ de 1993 del Ministerio de Salud de Colombia. El protocolo fue aprobado por los comités de investigación y ética de la Fundación Universitaria Sanitas y de las Clínicas Colsanitas.

\section{Resultados}

En total, se incluyeron en el estudio 94 pacientes mayores de 18 años con diagnóstico basal de adenocarcinoma gástrico avanzado; 64 pacientes eran de sexo masculino $(68,09 \%)$ y el promedio de edad de todos los pacientes fue de 59,85 años (desviación estándar, DE=12,69). El rango de edad fue de 26 a 83 años. Las mujeres tenían una edad promedio mayor que la de los hombres; sin embargo, esta diferencia no fue estadísticamente significativa ( $\mathrm{p}=0, \mathrm{IIO})$.

Según la clasificación histológica de Lauren, el tipo de cáncer más frecuentemente observado $(54,26 \%)$ en la población de estudio fue el difuso (tabla I).

La primera prueba diagnóstica evaluada fue la TC de abdomen con contraste. En los pacien- 
Tabla 1. Características basales

\begin{tabular}{|c|c|c|c|}
\hline Variable & \multicolumn{2}{|c|}{ Valor } & p \\
\hline Pacientes & & 94 & \\
\hline Sexo, H:M & & $64: 30$ & \\
\hline Edad (años) & Promedio ( $\pm \mathrm{DE})$ & (Mínimo-máximo) & \\
\hline Hombre & $58,71( \pm 11,88)$ & $(26-79)$ & $0,110^{*}$ \\
\hline Mujer & $62,26( \pm 14,18)$ & $(32-83)$ & \\
\hline $\begin{array}{l}\text { Clasificación histológica del cáncer gástrico } \\
\text { - Lauren }\end{array}$ & n (\%) & $\mathrm{IC}_{95 \%}$ & \\
\hline Tipo intestinal & $43(45,74)$ & $(36,04-55,78)$ & \\
\hline Tipo difuso & $51(54,26)$ & $(44,22-63,96)$ & \\
\hline
\end{tabular}

DE: desviación estándar; $\mathrm{IC}_{95 \%}$ : intervalo de confianza del $95 \%$

* Prueba no paramétrica U de Mann-Whitney

tes incluidos en este estudio, se reportó tumor primario $\mathrm{T}_{3}$ en el $56,38 \%$ de los exámenes de imágenes. No se informó compromiso ganglionar en el 55,32 \% y la categoría de metástasis más frecuente $(97,87 \%)$ fue la $\mathrm{M}_{\mathrm{o}}$, dado que los pacientes con diagnóstico de carcinomatosis peritoneal por TC fueron excluidos del estudio, a excepción de dos casos sometidos a laparoscopia diagnóstica con TC positiva para carcinomatosis para evaluar la posibilidad de practicar una cirugía oncológica peritoneal. Finalmente, la localización anatómica más frecuente $(47,87 \%)$ fue el cuerpo gástrico (tabla 2).

El segundo procedimiento de diagnóstico analizado fue la laparoscopia de estadificación. El tumor primario $\mathrm{T}_{3}$ fue el hallazgo reportado con mayor frecuencia $(43,62 \%)$ tras el procedimiento laparoscópico. En $56,38 \%$ de los pacientes se informó compromiso ganglionar y, en II,70 \%, carcinomatosis $M_{1}$ (tabla 3 ).

\section{Discusión}

Según los resultados de este estudio y de acuerdo con las incidencias reportadas para Colombia, hay un mayor porcentaje de hombres afectados por cáncer gástrico. Predominaron los tumores difusos, que son los de peor pronóstico oncológico, con el 54,36\%. En este grupo, el 56,38\% fueron tumores $\mathrm{T}_{3,}$ según la TC, y el 43,62\%, según la laparoscopia.
Tabla 2. Reporte de la tomografía computarizada

\begin{tabular}{|c|c|c|}
\hline \multirow{2}{*}{ Variable } & \multicolumn{2}{|c|}{ Valor } \\
\hline & $n(\%)$ & $I_{95 \%}$ \\
\hline \multicolumn{3}{|c|}{ Tumor primario $(\mathrm{T})$} \\
\hline $\mathrm{T}_{2}$ & $29(30,85)$ & $(22,42-49,79)$ \\
\hline $\mathrm{T}_{3}$ & $53(56,38)$ & $(46,30-65,96)$ \\
\hline $\mathrm{T}_{4}$ & $12(12,77)$ & $(7,45-21,00)$ \\
\hline \multicolumn{3}{|c|}{ Ganglios linfáticos (N) } \\
\hline Ausente & $52(55,32)$ & $(45,26-64,96)$ \\
\hline Presente & $42(44,68)$ & $(35,04-54,74)$ \\
\hline \multicolumn{3}{|l|}{ Metástasis (M) } \\
\hline $\mathrm{M}_{0}$ & $92(97,87)$ & $(92,57-99,41)$ \\
\hline $\mathrm{M}_{1}$ & $2(2,13)$ & $(0,58-7,42)$ \\
\hline \multicolumn{3}{|l|}{ Estadio } \\
\hline I & $42(44,68)$ & $(35,04-54,74)$ \\
\hline II & $40(42,55)$ & $(33,04-52,65)$ \\
\hline III & $10(10,64)$ & $(5,88-18,48)$ \\
\hline IV & $2(2,13)$ & $(0,58-7,42)$ \\
\hline \multicolumn{3}{|c|}{ Localización anatómica } \\
\hline Corporal & $45(47,87)$ & $(38,06-57,85)$ \\
\hline Antro & $15(15,96)$ & $(9,91-24,67)$ \\
\hline Antropilórica & $14(14,89)$ & $(9,08-23,46)$ \\
\hline Subcardial & $10(10,64)$ & $(5,88-19,12)$ \\
\hline Cuerpo-antro & $7(7,45)$ & $(3,65-14,58)$ \\
\hline Sin datos & $2(2,13)$ & $(0,58-7,42)$ \\
\hline Pilórica & $1(1,06)$ & $(0,18-5,78)$ \\
\hline
\end{tabular}


Tabla 3. Reporte de la laparoscopia de estadificación

\begin{tabular}{lcc}
\hline Variable & Valor & \\
\hline Tumor primario $(\mathrm{T})$ & $\mathbf{n ~ ( \% )}$ & $\mathbf{I C 9 5 \%}$ \\
$\mathrm{T}_{2}$ & $20(21,28)$ & $(14,22-30,59)$ \\
$\mathrm{T}_{3}$ & $41(43,62)$ & $(34,04-53,70)$ \\
$\mathrm{T}_{4}$ & $33(35,11)$ & $(26,22-45,17)$ \\
Ganglios linfáticos $(\mathrm{N})$ & & \\
Ausente & $41(43,62)$ & $(34,04-53,70)$ \\
Presente & $53(56,38)$ & $(46,3-65,96)$ \\
Metástasis (M) & & \\
$\mathrm{M}_{0}$ & $83(88,30)$ & $(80,25-93,34)$ \\
$\mathrm{M}_{1}$ & $11(11,70)$ & $(6,66-19,75)$ \\
\hline
\end{tabular}

El porcentaje de enfermedad ganglionar no presentó diferencias estadísticamente significativas: fue de $55,32 \%$ con la TC y de $56,38 \%$ con la laparoscopia; en el grupo de pacientes que la padecían, se encontraron II (II, 7 \%) con enfermedad metastásica, la cual no fue documentada en la TC. Esto modificó la conducta en aquellos pacientes que, según la TC, tenían una enfermedad resecable, hasta que la laparoscopia demostró lo contrario. Esto concuerda con lo encontrado en una revisión sistemática sobre la precisión de la laparoscopia diagnóstica en el cáncer gástrico, la cual puede modificar el manejo en 8,5 a 59,6 \% de los pacientes ${ }^{6,7}$.

Teniendo presente que los pacientes con enfermedad metastásica diagnosticada por TC no fueron incluidos en este estudio, fue posible documentar enfermedad peritoneal que no había sido evidente en las imágenes en II,7\% de los casos. En la literatura científica mundial, se reporta una sensibilidad del $25 \%$ y una especificidad del $9 \%$ para la TC.

Si bien este estudio no es suficiente para detectar o excluir enfermedad peritoneal en aquellos pacientes con cáncer gástrico, es muy específica y presenta un bajo porcentaje de falsos positivos, los cuales pueden ser reclasificados eventualmente mediante laparoscopia ${ }^{9}$.

Con respecto al papel del lavado peritoneal, se han reportado recurrencias tumorales del II,I al
IOo \% en los pacientes con lavado positivo y, del o al 5I \% en aquellos con lavado negativo ${ }^{\text {Io }}$. En un estudio japonés de 2007 , se reportó detección de células libres en $29 \%$ de los casos ${ }^{\text {II }}$. Los pacientes con lavado peritoneal positivo tienen un pronóstico que se acerca más al de los tumores en estadio IV que al de los tumores en estadio III, a pesar de no haber enfermedad peritoneal evidente en la laparoscopia. En nuestra experiencia, aunque en este estudio el lavado peritoneal fue positivo en un porcentaje que puede considerarse bajo, no deja de ser una estrategia costo-efectiva, que no prolonga el tiempo quirúrgico ni agrega morbilidad al procedimiento, lo que hace viable su utilización rutinaria.

En nuestro medio, la laparoscopia de estadificación es una estrategia rentable con un impacto positivo en el manejo integral de los pacientes con cáncer gástrico, acorde a lo que se reporta en la literatura científica mundial ${ }^{12,13}$.

\section{Conclusiones}

Los hallazgos del presente estudio confirman lo descrito en la literatura científica sobre la estadificación preoperatoria del cáncer gástrico. Si se dispone de un servicio de radiología experimentado, es posible tener una alta tasa de detección de enfermedad metastásica y, aun así, con la laparoscopia se puede detectar hasta un II \% de pacientes con enfermedad peritoneal que no es evidente en la TC.

Con estos resultados, podemos recomendar utilizar la laparoscopia de estadificación en todos los pacientes con cáncer gástrico avanzado cuya TC abdominal sea negativa para enfermedad peritoneal, con el fin de detectar los falsos negativos y lograr, así, un impacto en el manejo integral del cáncer gástrico, evitando, sobre todo, la morbilidad de una cirugía mayor en los casos de enfermedad irresecable y siempre procurando obtener los mejores resultados oncológicos posibles.

Financiación. Se declara que los recursos de financiación del proyecto provienen en su totalidad de aportes de los autores del proyecto de investigación 
Conflicto de interés. No hubo conflictos de ninguna naturaleza para el desarrollo de la investigación.

\section{Referencias}

I. Howlader N, Noone AM, Krapcho M, Miller D, Bishop $\mathrm{K}$, Altekruse SF, et al. SEER Cancer Statistics Review, I975-20I3. Bethesda: National Cancer Institute; 20I5. Fecha de consulta: 20 de noviembre de 20I6. Disponible en: http://seer.cancer.gov/csr/1975_2013/

2. Mehmedovic A, Mesihovic R, Saray A, Vanis N. Gastric cancer staging: EUS and CT. Med Arch. 2014;68:34-6.

3. Correa P. Cáncer gástrico: una enfermedad infecciosa. Rev Colomb Cir. 20II;26:III-7.

4. International Agency for Research on Cancer. The GLOBOCAN Project. 20I2 Fecha de consulta: 20 de noviembre de 20I6. Disponible en: http://globocan.iarc. fr/Default.aspx.

5. Karanicolas PJ, Elkin EB, Jacks LM, Atoria CL, Strong VE, Brennan MF, et al. Staging laparoscopy in the management of gastric cancer: A population based analysis. J Am Coll Surg. 20II;213:644-5I.

6. Kwee RM, Kwee TC. Modern imaging techniques for preoperative detection of distant methastases in gastric cancer. World J Gastroenterol. 2015;21:10502-9.
7. Leake PA, Cardoso R, Seevaratnam R, Lourenco L, Helyer L, Mahar A, et al. A systematic review of the accuracy and indications for diagnostic laparoscopy prior to curative-intent resection of gastric cancer. Gastric Cancer. 2012;15(Suppl.):S38-47.

8. Shah MA. Gastric cancer: An update. Curr Oncol Rep. 2006;8:I83-9I.

9. Burbidge S, Mahady K, Naik K. The role of CT and staging laparoscopy in the staging of gastric cancer. Clin Radiol. 20I3;68:25I-5.

Io. Leake PA, Cardoso R, Seevaratnam R, Lourenco L, Helyer L, Mahar A, et al. A systematic review of the accuracy and utility of peritoneal cytology in patients with gastric cancer. Gastric Cancer. 2012;15(Suppl.I):27-37. doi: I0.IOO7/sIOI2O-OII-007I-Z

II. Nakagawa S, Nashimoto A, Yabusaki H. Role of staging laparoscopy with peritoneal lavage cytology in the treatment of locally advanced gastric cancer. Gastric Cancer. 2007;IO:29-34.

I2. Convie L, Thompson RJ, Kennedy R, Clements WDB, Carey PD, Kennedy JA. The current role of staging laparoscopy in oesophagogastric cancer. Ann R Coll Surg Engl. 2015;97:I46-50.

I3. Bhatti AB, Haider S, Khattak S, Syed AA. Staging laparoscopy in gastroesophageal and gastric adenocarcinoma: First experience from Pakistan. Indian J Cancer. 20I4; 5I:15-7. 